\title{
Validation of END-of-life ScorlNG-system to identify the dying patient: a prospective analysis
}

Gianluca Villa ${ }^{1,2^{*}}$, Timothy Amass ${ }^{3}$, Rosa Giua ${ }^{2}$, lacopo Lanini ${ }^{1}$, Cosimo Chelazzi ${ }^{2}$, Lorenzo Tofani ${ }^{1}$, Rory McFadden ${ }^{4}$, A. Raffaele De Gaudio ${ }^{1,2}$, Sean OMahony ${ }^{4}$, Mitchell M. Levy ${ }^{3}$ and Stefano Romagnoli, ${ }^{1,2}$

\begin{abstract}
Background: The "END-of-Life ScorlNG-System" (ENDING-S) was previously developed to identify patients at highrisk of dying in the ICU and to facilitate a practical integration between palliative and intensive care. The aim of this study is to prospectively validate ENDING-S in a cohort of long-term critical care patients.

Materials and methods: Adult long-term ICU patients (with a length-of-stay> 4 days) were considered for this prospective multicenter observational study. ENDING-S and SOFA score were calculated daily and evaluated against the patient's ICU outcome. The predictive properties were evaluated through a receiver operating characteristic (ROC) analysis.

Results: Two hundred twenty patients were enrolled for this study. Among these, 21.46\% died during the ICU stay. ENDING-S correctly predicted the ICU outcome in $71.4 \%$ of patients. Sensitivity, specificity, positive and negative predictive values associated with the previously identified ENDING-S cut-off of 11.5 were $68.1,72.3,60$ and 89.3\%, respectively. ROC-AUC for outcome prediction was 0.79 for ENDING-S and 0.88 for SOFA in this cohort.
\end{abstract}

Conclusions: ENDING-S, while not as accurately as in the pilot study, demonstrated acceptable discrimination properties in identifying long-term ICU patients at very high-risk of dying. ENDING-S may be a useful tool aimed at facilitating a practical integration between palliative, end-of-life and intensive care.

Trial registration: Clinicaltrials.gov Identifier: NCT02875912; First registration August 4, 2016.

Keywords: End of life, Scoring system, palliative care, intensive care unit

\section{Background}

The advanced technological treatments available in the intensive care unit (ICU) aim at managing acute illness and at the same time supporting multiorgan failure [1]. Considering an average mortality rate of $20 \%$ among critical care patients, only $80 \%$ of ICU patients really

\footnotetext{
* Correspondence: gianluca.villa@unifi.it

${ }^{1}$ Section of Anesthesiology, Intensive Care and Pain Therapy, Departmnt of Health Sciences, University of Florence, Florence, Italy

${ }^{2}$ Department of Anesthesia and Intensive Care, Azienda Ospedaliero-Universitaria Careggi, Largo Brambilla,3, 50134 Florence, Italy Full list of author information is available at the end of the article
}

benefit from these highly expensive treatments [1]; furthermore, a not irrelevant percentage of ICU survivors will die in any case before hospital discharge [2]. Given the likelihood of morbidity or mortality, palliative and end-of-life care should be routinely considered in the ICU [3]. As such, the World Health Organization has described palliative care as "an approach that improves the quality of life of patients and their families facing the problems associated with life-threatening illness" [4]. The physician should be thus aware of the patient and family needs to improve the management of physical, psychological and spiritual symptoms. Additionally,

(c) The Author(s). 2020 Open Access This article is licensed under a Creative Commons Attribution 4.0 International License, which permits use, sharing, adaptation, distribution and reproduction in any medium or format, as long as you give appropriate credit to the original author(s) and the source, provide a link to the Creative Commons licence, and indicate if changes were made. The images or other third party material in this article are included in the article's Creative Commons licence, unless indicated otherwise in a credit line to the material. If material is not included in the article's Creative Commons licence and your intended use is not permitted by statutory regulation or exceeds the permitted use, you will need to obtain permission directly from the copyright holder. To view a copy of this licence, visit http://creativecommons.org/licenses/by/4.0/ The Creative Commons Public Domain Dedication waiver (http://creativecommons.org/publicdomain/zero/1.0/) applies to the data made available in this article, unless otherwise stated in a credit line to the data. 
being aware of the value of palliative care services to help meet the families' and patients' needs and align therapy to the prognosis of the patient balanced with their preferences and values $[4,5]$.

Beyond those clearly at the final stages of irreversible diseases (i.e. end-of-life), all patients admitted in the ICU require an early integration between a comprehensive palliative approach and intensive care treatment $[4$, 6]. The probability of dying of each critical care patient should be evaluated early on at the ICU admission and continuously reassessed during the entire ICU length of stay; the amount of palliative care treatments should thus be integrated accordingly [4].

Although excellent scoring systems are available in the ICU for prognostic and clinical monitoring purposes (e.g. Acute Physiologic Assessment and Chronic Health Evaluation, APACHE, and Sequential Organ Failure Assessment score, SOFA [7]), an accurate identification of end-of-life patients in the ICU is still cumbersome [810]. As example, the usefulness of several scores (e.g. the APACHE score) is mainly validated at the ICU admission, when the patients' responsiveness to intensive care treatments is not clear yet. Other scoring systems (e.g. the SOFA score) are well validated to monitor the organ dysfunctions over time in the ICU; nevertheless, the requirement of biochemical data for the scoring calculation may limit their routine use/daily application [7]. Finally, the requirement for, and to what degree, palliative care should be integrated with intensive care for all ICU patients is often not objectively defined and instead determined by individual physician's perspective $[11,12]$.

The "END-of-Life ScorING-System" (ENDING-S) was previously developed to: 1 ) identify patients at very high risk of dying in the ICU and 2) facilitate a practical integration between palliative, end-of-life and intensive care treatments [11]. In a pilot study, ENDING-S presented acceptable calibration and discrimination properties in identifying patients at very high risk of dying in the ICU, with a receiver operating characteristic-area under the curve (ROC-AUC) analysis equal to 0.98 (95\%CI, 0.97 to 1) and agreement between the predicted probability and the observed frequency of death in the ICU $(p>0.05$ at HosmerLemeshow test) were preliminarily observed [11].

The aim of this observational study is to prospectively validate ENDING-S in a cohort of critical care patients with an ICU length of stay longer than 4 days.

\section{Methods}

This observational prospective study was performed in three ICUs: Rhode Island Hospital's medical ICU (Providence RI), Rush Medical Center's medical ICU (Chicago IL) and Azienda Ospedaliera Universitaria Careggi's surgical and medical ICU (Florence Italy). The institutional review boards of each center reviewed and approved the protocol (clinicaltrials.gov Identifier: NCT02875912; first retrospective registration: August 4, 2016). Written consent for analysis and publication of clinical data was obtained from all consentable patients. If the patient was not able to sign consent forms at the study enrollment, permission for analysis and publication of clinical data was obtained from a surrogate or waived in accordance with local ethics committee.

All adult patients admitted in the ICU from September 2015 to March 2017 were considered eligible for the study. Patients admitted to the ICU for end-of-life care were excluded. In order to consider only the long-term ICU patients, those with an ICU length of stay shorter than 4 days were excluded from the analysis. Data abstraction forms were prospectively completed for all eligible patients. In particular, ENDING-S and SOFA scores were calculated daily from the ICU day 4 to the ICU discharge for each enrolled patient. In accordance with the previous paper [1], ENDING-S score was calculated as:

$$
\begin{aligned}
\text { ENDING-S }= & (7.25 \cdot \text { Days of MV } / \text { ICU LoS }) \\
& +(10.45 \cdot \text { Days of Vasoactive drugs } / \mathrm{ICU} \text { LoS }) \\
& +(3 \cdot \text { Sepsis })+(0.3 \cdot \mathrm{ICU} \text { LoS }) .
\end{aligned}
$$

where Days of MV/ICU LoS expresses the ratio between the current days in which the patient requires mechanical ventilation (MV) and the current ICU length of stay (LoS, quantitative variable), Days of Vasoactive drugs/ICU LoS expresses the ratio between the current days in which the patient requires vasoactive drugs and the current ICU length of stay (quantitative variable), Sepsis expresses a septic condition (dichotomous variable: 1 if currently affected, otherwise 0), ICU LoS expresses the current ICU length of stay (quantitative variable).

The ICU patients' management has not changed according to the value of ENDING-S observed.

According to the patients' outcome, the enrolled population was divided into two groups, either "survived" or "died". Death in the ICU being used as a surrogate to identify those ICU patients that had been at the end of their life prior to death. For "died" patients, maximization of palliative care and end-of-life care are thus certainly required. For "survived" patients, a certain form of palliative care is required according to the patients' multidimensional evaluation and expected prognosis, but very unlikely these patients require end-of-life care.

The association between the daily values of ENDING$\mathrm{S}$ and patients' outcome at the ICU discharge was tested through a logistic regression analysis (OR, 95\% CI). The ENDING-S predictive properties were evaluated with a ROC analysis. The effectiveness of ENDING-S cut-off 11.5 was previously validated for prediction of death during the ICU stay. The positive predictive value (PPV), 
negative predictive values (NPV), sensitivity and specificity of ENDING-s were calculated.

Similarly, the association between the daily values of SOFA score and patients' outcome was tested through a logistic regression analysis. The effectiveness of SOFA score was assessed for prediction of death during the ICU stay. In the absence of a specific cut-off point, only a ROC analysis was performed for this scoring system.

Continuous parameters observed in the population are reported as median [interquartile range] or mean \pm standard deviation (SD), where appropriate; dichotomous parameters are expressed as crude number and percentage. A $p$ value of 0.05 has been considered for statistical significance.

Data was analyzed using STATA 9.1 software (STATA corp, 490, Lakeway Drive College Station, 77,845, Texas, US).

\section{Results}

Nine hundred and eleven patients were prospectively screened for this multicenter study. Among these, 220 patients had an ICU length of stay longer than 4 days and thus prospectively enrolled and considered for the analysis. The enrollment procedures are reported in Fig. 1.
Among the enrolled patients, $21.46 \%(47 / 220)$ died during the ICU stay, while $78.64 \%(173 / 220)$ survived and were discharged from the ICU. The patients' characteristics, for both survivor and death groups, are reported in Table 1.

Among patients who died, 32 of $47(68.09 \%)$ had ENDING-S values higher than 11.5 (true positive), while the remaining 15 (31.91\%) had at least an ENDING-S < 11.5 during the ICU stay (false negative). However, among patients who survived to ICU discharge, 125 of 173 (72.25\%) had ENDING-S values smaller than 11.5 (true negative), while the remaining $48(27.75 \%)$ had at least an ENDING-S $\geq 11.5$ during the ICU stay (false positive). Given these characteristics, ENDING-S correctly predicted for 157 patients $(157 / 220,71.4$ 95\%CI [0.65-0.77] and was statistically associated with the patients' ICU outcome, $p<0.0001$, OR 1.182, 95\%CI [1.115-1.253].

Sensitivity and specificity associated with ENDING$S$ cut-off of 11.5 were 68.09 and $72.25 \%$, respectively; positive and negative predictive values were 60 and $89.28 \%$, respectively. ROC-AUC of 0.79 was found for ENDING-S in this validation set (Fig. 2, Panel a), 95\% CI [0.71-0.86].

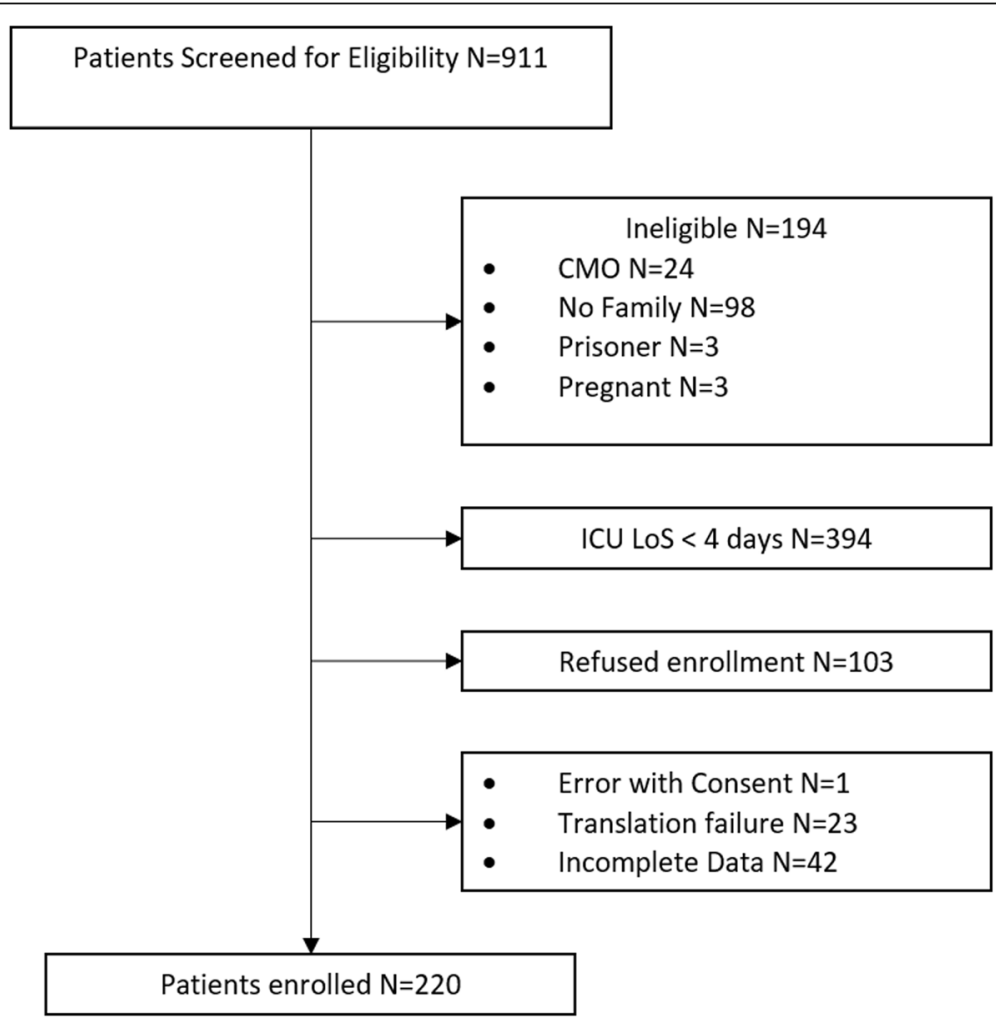

Fig. 1 The enrollment process. Over the entire population potentially eligible for this prospective study, 194 patients were excluded because admitted in the ICU for comfort measure only (CMO), for lack of family members (required for the qualitative analysis of the study, data not presented), or because pregnant or prisoner. In order to consider only "long term" ICU patients, those with an ICU length of stay (LoS) $<4$ days were excluded. Finally, 103 patients refused to be enrolled in this observational study, forms were not completed for 43 patients ( 1 consent form and 42 clinical data forms) and 23 patients were excluded because not English or Italian native speaking 
Table 1 Patients' characteristics at the ICU admission and at discharge from the ICU

\begin{tabular}{|c|c|c|c|c|}
\hline & $\begin{array}{l}\text { All } \\
(\boldsymbol{n}=220)\end{array}$ & $\begin{array}{l}\text { Death } \\
(n=47)\end{array}$ & $\begin{array}{l}\text { Survivor } \\
(n=173)\end{array}$ & $p$ \\
\hline Age (yrs) & $64.5 \pm 16.8$ & $66.1 \pm 17.1$ & $64.1 \pm 16.6$ & 0.12 \\
\hline Sex & & & & 0.41 \\
\hline Male & $128(58.1 \%)$ & $30(63.8 \%)$ & $98(56.6 \%)$ & \\
\hline Female & $92(41.9 \%)$ & $17(36.2 \%)$ & 75 (43.4\%) & \\
\hline Race & & & & 0.04 \\
\hline White & $181(82.3 \%)$ & $34(72.3 \%)$ & $147(84.9 \%)$ & \\
\hline Black or African American & $12(5.4 \%)$ & $6(12.8 \%)$ & $6(3.5 \%)$ & \\
\hline Other & $27(12.3 \%)$ & $7(14.9 \%)$ & $20(11.6 \%)$ & \\
\hline Country of birth & & & & 0.06 \\
\hline Italy & 119 (54\%) & 19 (38.4\%) & $101(58.3 \%)$ & \\
\hline USA & $76(34.3 \%)$ & $22(46.8 \%)$ & $54(31.1 \%)$ & \\
\hline Other & $1(0.4 \%)$ & $0(0 \%)$ & $1(0.6 \%)$ & \\
\hline Admission source & & & & 0.15 \\
\hline Home & $54(24.3 \%)$ & $14(29.8 \%)$ & $40(23.1 \%)$ & \\
\hline Nursing home/Skilled nursing facility & $13(5.9 \%)$ & $5(10.6 \%)$ & $8(4.6 \%)$ & \\
\hline Acute care facility/Outside hospital & $153(69.8 \%)$ & $28(59.6 \%)$ & $125(72.3 \%)$ & \\
\hline SOFA score at ICU admission & $5.4 \pm 3.4$ & $8.6 \pm 3.6$ & $4.8 \pm 2.9$ & $<0.01$ \\
\hline Vasoactive medication requirements at ICU admission & $30(13.6 \%)$ & $10(21.3 \%)$ & $20(11.6 \%)$ & 0.09 \\
\hline $\mathrm{PaO2} / \mathrm{FiO2} \leq 300$ at ICU admission & $44(20 \%)$ & $17(36.2 \%)$ & $27(15.6 \%)$ & $<0.01$ \\
\hline Serum creatinine $\geq 2 \mathrm{mg} / \mathrm{dl}$ at ICU admission & $23(10.5 \%)$ & $10(21.3 \%)$ & $13(7.5 \%)$ & 0.01 \\
\hline Serum bilirubin $\geq 2 \mathrm{mg} / \mathrm{dl}$ at ICU admission & $5(2.3 \%)$ & $3(6.4 \%)$ & $2(1.1 \%)$ & 0.07 \\
\hline Length of ICU stay (days) & $10.3 \pm 6.6$ & $11.6 \pm 6.4$ & $9.9 \pm 6.9$ & 0.07 \\
\hline Status change in DNR/DNI/CMO & & & & $<0.01$ \\
\hline Yes & $55(25.7 \%)$ & $41(87.2 \%)$ & $14(8.1 \%)$ & \\
\hline No & $165(74.3 \%)$ & $6(12.8 \%)$ & $159(91.9 \%)$ & \\
\hline After discharge & & & & NA \\
\hline Another hospital floor & & & $155(89.6 \%)$ & \\
\hline Nursing home & & & $2(1.2 \%)$ & \\
\hline Long-term ventilator assist facility & & & $5(2.9 \%)$ & \\
\hline Home & & & $3(1.7 \%)$ & \\
\hline Other & & & $8(4.6 \%)$ & \\
\hline
\end{tabular}

Abbreviations: DNI/DNR/CMO Do not intubate/Do not resuscitate/Comfort measure only

Considering daily values of SOFA score both for survived and not survived patients, a mean SOFA score of $8.4 \pm 4.1$ was observed for patients who died, while $4 \pm$ 2.6 was observed for patients who survived at the ICU discharge. A ROC-AUC of 0.88 (95\%CI $0.81-0.96)$ was found for daily values of SOFA score in predicting ICU death (Fig. 2, Panel b).

\section{Discussion}

In this observational study, the previously defined ENDING-score was prospectively tested in a cohort of critical care patients with an ICU length of stay longer than 4 days in order to validate its discriminative effect in identifying patient at very high risk of dying in the ICU.

The comparison between the ICU outcome observed for every enrolled patient and that expected according to the ENDING-S cut-off of 11.5 reveals a direct association between ENDING-S and patients' ICU outcome $p<0.0001)$. In particular, every incremental increase in ENDING-S value increased the OR estimate of death in the ICU by 1.18 .

Compared with discrimination properties showed by ENDING-S in the pilot study, less efficient characteristics were observed in this prospective validation. Indeed, a ROC-AUC of 0.98 (95\%CI, 0.97-1.00) was observed in 

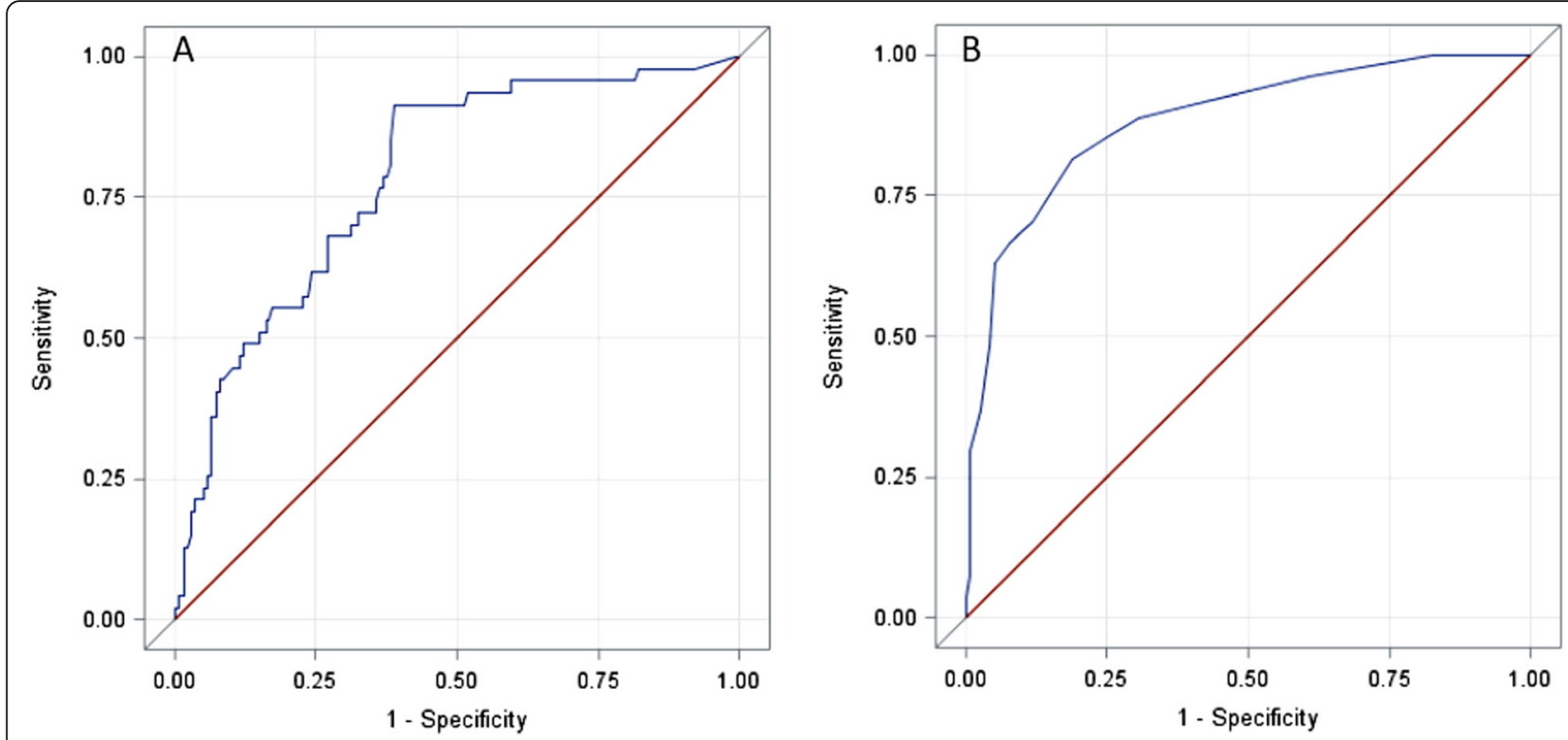

Fig. 2 Likelihood of ICU Death. ROC curves for patients' outcome discrimination for both ENDING-S (Panel a, ROC-AUC 0.79) and SOFA score (Panel b, ROC-AUC 0.88)

the calibration set of the pilot study and further confirmed in the internal validation performed in the same study (ROC-AUC 0.98; 95\%CI 0.96-1.00). On the other hand, a ROC-AUC of 0.79 (95\%CI 0.71-0.86) was observed in this prospective external validation. This effect was expected considering that a cross-validation test was applied in the pilot study to internally validate the model and to assess the predictive properties of ENDING-S. Although statistically correct, the use of an internal validation might have optimistically confirmed the preliminary results, overestimating the ENDING-s performance characteristics. Nevertheless, a ROC-AUC equal to 0.79 is still sufficient to confirm the acceptable discrimination properties of ENDING-S in identifying patients at very high risk of dying in the ICU.

Similar to the ROC-AUC, the sensitivity and specificity values previously observed in the pilot study are reduced in this external validation study [11].

The identification of end-of-life patients is quintessential for an adequate integration between qualitative and intensive care treatments during the ICU stay $[13,14]$. Notably, palliative care should not be considered as an alternative for the intensive care in these patients; this care should instead be concomitantly made available for the patients and their family early on from the ICU admission [15]. The importance of palliative care with respect to intensive care should be proportional with the probability that the patient is at very high risk of dying in the ICU and in accordance with the specific patient and family needs.

Identification of the patient at very high risk of dying is not the only limitation for palliative care integration in the ICU [16]. Indeed, the "relative amount" of palliative care that should be considered adequate for a specific patient in a specific moment during the ICU stay is difficult to define. Most of data in literature show that the management of palliative and end-of-life care is still determined by the physician's subjective experiences, religion, level of expertise and other nonobjective and unquantifiable variables $[1,15,17-19]$. Used beside other tools and comprehensive clinical evaluations, this objective tool, able to accurately identify dying patients and to suggest the adequate ratio between qualitative and intensive care for those patients at very high-risk of dying in the ICU, might help the physician appropriately integrate palliative care in the ICU [9].

Assuming that the progression toward end-of-life should be characterized by an increasing presence of palliative care in the global management of the patient, the probability of being at very high risk of death in the ICU can be used as an indicator of the percentage need of palliative and mainly end-of-life care integration. Treatments based on patients' and families' needs (i.e. targeted on communication, psychological, social personal and/or spiritual well-being), should be progressively prioritized within the efforts of the health care team. In these terms, evaluating the ENDING-S and SOFA score might also be potentially useful for guiding palliative care integration in the ICU (Fig. 3).

While SOFA does outperform ENDING-S, ENDING-S may still be considered clinically useful when compared to a SOFA score in two ways. First, the negative predictive value of ENDING-S of $89.28 \%$ suggests that an ENDING-S score less than 11.5 is clinically valuable in 


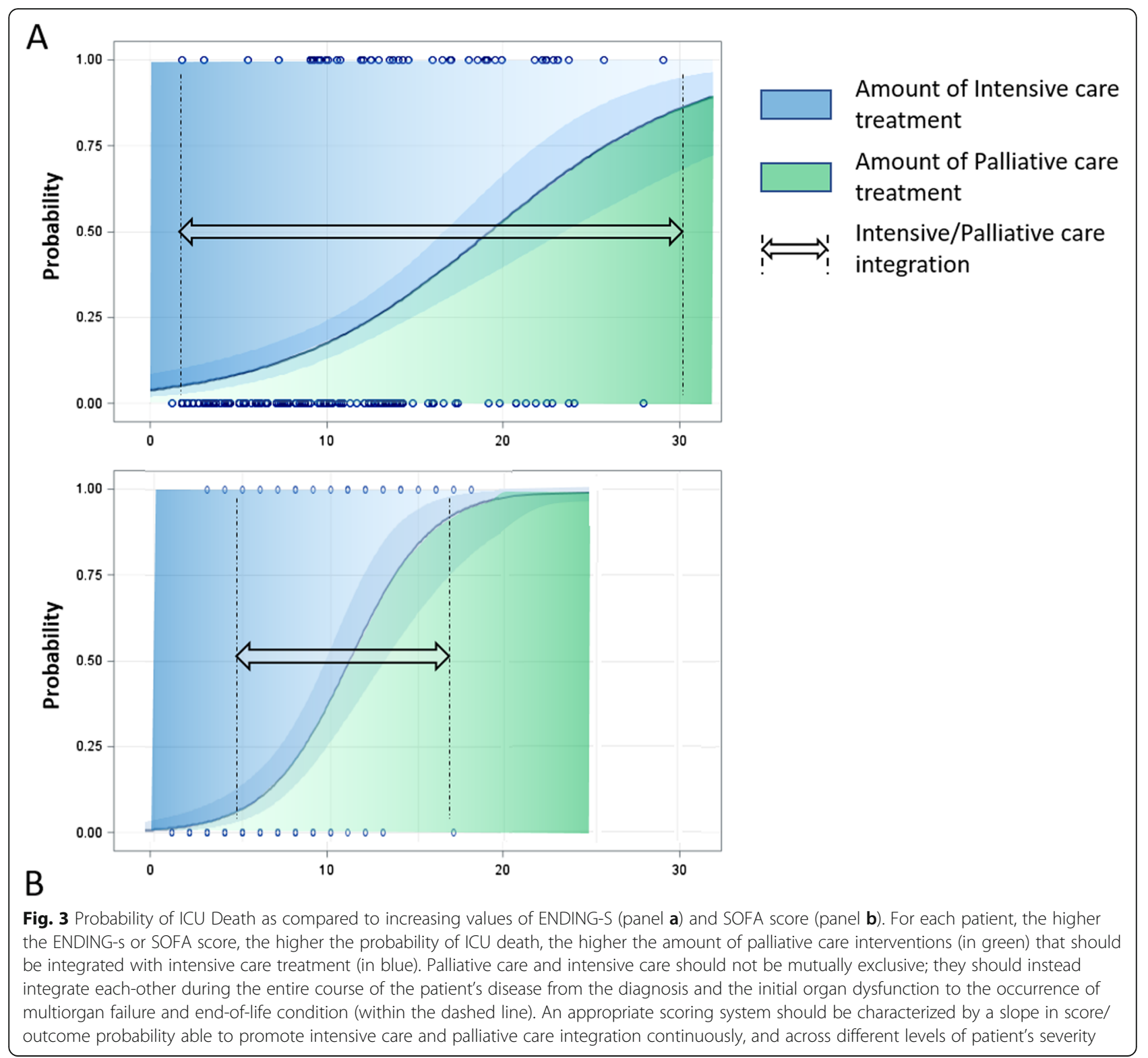

aiding clinicians in identifying patients that are likely to survive. Combining this with the fact that an ENDING-S score does not require any laboratory data as the SOFA score does, ENDING-S can be calculated every day, easily, on each patient. SOFA score requires laboratory data which was often missing in this cohort limiting the usefulness of a SOFA score in providing daily prognostic information.

Another important difference between ENDING-S and SOFA score is in the variation during the ICU length of stay of clinically stable patients who spend their end-oflife period in the ICU before dying. Although in critical condition, several patients have high and unchanged values of SOFA score over time, specifically before dying. Thus, a prognostic tool only based on SOFA score would not change, failing to alert the physician to the increasing amount of palliative and end-of-life care required for that patient. On the other hand, as ENDING$\mathrm{S}$ is influenced by the progression of days spent in the ICU, it increases over time even in absence of clinical changes. This suggests that, by virtue of staying in the ICU, patients could require a progressively increasing amount of qualitative care over time.

There are several limitations in this study; among these, the use of ICU mortality as a surrogate to identify end-of-life is the most important. The aim of ENDING$\mathrm{S}$ is to identify those patients likely being at very high risk of dying in the ICU and suggest the physician to appropriately consider palliative care integration for these patients. Interestingly, an objective definition of end-of- 
life status is still lacking in clinical practice, even for patients who are not hospitalized [8]. For this reason, as in other papers $[20,21]$, the outcome observed at the ICU discharge has been used as a surrogate for end-of-life condition for patients both enrolled in the pilot study and for those enrolled in this prospective validation. Notably, despite death in the ICU certainly being associated with the end-of-life of patients, ICU survival does not necessarily exclude the end-of-life condition or the requirement of palliative care [22]. Unfortunately, a systematic follow-up for patients discharged from the ICU is lacking in this study.

Another important conceptual limitation refers to the exclusive use of the patient's prognosis to guide the palliative care/intensive care integration. Several authors agree that palliative care should be based on needs of patients and their family instead of being exclusively based on patients' prognosis. A comprehensive qualitative evaluation should be integrated on ENDING-S parameters to further improve its role in guiding palliative care. Issues such as patients and family needs, perception of the disease, communication between family members and with health care providers should be considered and analyzed with this aim.

\section{Conclusion}

Although values observed in the pilot study resulted in a slightly overestimated prediction model, acceptable discrimination properties have been demonstrated for ENDING-S in identifying patients at very high risk of dying with an ICU stay longer than 4 days. Although SOFA score was more effective and accurate in predicting patients' death in the ICU, the ENDING-S score offers the benefit of not requiring laboratory data, a strong negative predictive value for ICU death, and daily changes by including length of stay. These factors may allow ENDING-S to be meaningfully integrated into daily practice and aid in the integration between palliative, end-of-life and intensive care. Nevertheless, further prospective validations and comparisons between EDNING-S and other standardized score are necessarily to better characterize the clinical application of this tool.

\section{Acknowledgements}

Not applicable.

\section{Authors' contributions}

G. V.: This author substantial helped to conception and design, acquisition of data, analysis and interpretation of data; T. A.: This author was accountable for all aspects of the work thereby ensuring that questions related to the accuracy or integrity of any part of the work were appropriately investigated and resolved; R. G.: This author helped to conception and design, acquisition of data; I. L.: This author helped to conception and design the study; C. C.: This author helped to draft the article or review it critically for important intellectual content; L. T.: This author helped to analysis and interpretation of data; R.M.F.: This author helped to draft the article or review it critically for important intellectual content; A. R. D. G.: This author gave substantial contributions to conception and design of this study and gave final approval of the version to be published. S.OM.: This author helped to draft the article or review it critically for important content; M. L.: This author gave substantial contributions to conception and design of this study and gave final approval of the version to be published. S. R.: This author helped to draft the article or review it critically for important intellectual content. The authors read and approved the final manuscript.

\section{Funding}

Ente Cassa di Risparmio di Firenze (grant number 2014.0679), the "Cox foundation" (grant number: NA), the "Philip and Irene Toll Gage Foundation" (grant number: NA) and the National Heart Lung and Blood Institute (NHLBI grant number: T32 HL134625) have economically supported the feasibility and management of this study through research grants aimed at study coordination, statistical analysis and recruitment of investigators for this study. The funders had no role in study design, data collection and analysis, decision to publish, or preparation of the manuscript.

\section{Availability of data and materials}

The datasets analysed during the current study are available from the corresponding author on reasonable request.

\section{Ethics approval and consent to participate}

The institutional review boards of each center reviewed and approved the protocol; in particular the "Comitato Etico Area Vasta Toscana Centro" approved the study protocol for the Azienda Ospedaliero Universitaria Careggi in Florence, the Internal Review Board of the Rhode Island Hospital approved the study protocol in Providence and the Internal Review Board of the Rush University Medical Center approved the study protocol in Chicago. (clinicaltrials.gov Identifier: NCT02875912). Written consent for analysis and publication of clinical data was obtained from all consentable patients. If the patient was not able to sign consent forms at the study enrollment, permission for analysis and publication of clinical data was obtained from a surrogate or waived in accordance with local ethics committee. All methods of consent were approved by the appropriate ethics committees.

\section{Consent for publication}

Not Applicable.

\section{Competing interests}

We have the following interests: GV received from Baxter honoraria for lectures, from Pall International support for travel expenses, hotel accommodations, and registration to meetings. SR received from Baxter, Orion Pharma, and Vygon honoraria for lectures, from ICU Medical, MSD and Medtronic grants for consultancy, form Baxter, BBraun, Pall International, and Vygon support for travel expenses, hotel accommodations, and registration to meetings. CC received support for meetings (travels, hotel accommodations, and/or registration) by BBraun, Astellas, MSD, Pfizer, Pall International, Baxter, and Orion Pharma, for lectures by Orion Pharma. ARDG received research grants from MSD Italia, Baxter, Pall international. There are no patents, products in development or marketed products to declare. This does not alter our adherence to all the journal policies.

\section{Author details}

'Section of Anesthesiology, Intensive Care and Pain Therapy, Departmnt of Health Sciences, University of Florence, Florence, Italy. ${ }^{2}$ Department of Anesthesia and Intensive Care, Azienda Ospedaliero-Universitaria Careggi, Largo Brambilla,3, 50134 Florence, Italy. ${ }^{3}$ Department of Medicine, Division of Pulmonary Critical Care \& Sleep, Brown University, Providence, RI, USA. ${ }^{4}$ Department of Internal Medicine, Palliative Medicine Section, Rush University Medical Center, Chicago, IL, USA.

Received: 24 November 2019 Accepted: 4 March 2020

Published online: 09 March 2020

\section{References}

1. Bertolini G, Boffelli S, Malacarne P, Peta M, Marchesi M, Barbisan C, et al. End-of-life decision-making and quality of ICU performance: an observational study in 84 Italian units. Intensive Care Med. 2010;36(9):1495504 Available from: http://www.ncbi.nlm.nih.gov/pubmed/20464541. [cited 2013 Dec 28]. 
2. Angus DC, Barnato AE, Linde-Zwirble WT, Weissfeld LA, Watson RS, Rickert T, et al. Use of intensive care at the end of life in the United States: An epidemiologic study*. Crit Care Med. 2004;32(3):638-43 Available from: http://content.wkhealth.com/linkback/openurl?sid=WKPTLP: landingpage\&an=00003246-200403000-00003. [cited 2014 Jun 30].

3. Orsi L, Gristina GR. The right choice at the right time. Minerva Anestesiol. Italy. 2017:83(5):436-9.

4. Gristina GR, De Gaudio R, Mazzon D, Curtis JR. End of life care in Italian intensive care units: where are we now? Minerva Anestesiol. 2011;77(9):91120 Available from: http://www.ncbi.nlm.nih.gov/pubmed/21878873.

5. Amass TH, Villa G, OMahony S, Badger JM, McFadden R, Walsh T, et al. Family Care rituals in the ICU to reduce symptoms of post-traumatic stress disorder in family members-a multicenter, multinational, before-and-after intervention trial. Crit Care Med United States. 2020;48(2):176-84.

6. Vuong C, Kittelson S, McCullough L, Yingwei Y, Hartjes T. Implementing primary palliative care best practices in critical care with the Care and Communication Bundle. BMJ open Qual. 2019;8(3):e000513.

7. Giannoni C, Chelazzi C, Villa G, De Gaudio AR. Organ dysfunction scores in ICU. Trends Anaesth Crit Care. 2013;3(3):89-96 Available from: http:// linkinghub.elsevier.com/retrieve/pii/S221084401300021X. [cited 2013 Sep 15]. Elsevier Ltd.

8. Luce JM. End-of-life decision making in the intensive care unit. Am J Respir Crit Care Med. 2010;182(1):6-11. Available from: http://www.ncbi.nlm.nih. gov/pubmed/20194809. [cited 2012 Nov 9].

9. Fischer SM, Gozansky WS, Sauaia A, Min S-J, Kutner JS, Kramer A. A practical tool to identify patients who may benefit from a palliative approach: the CARING criteria. J Pain Symptom Manage. 2006;31(4):285-92 Available from: http://www.ncbi.nlm.nih.gov/pubmed/16632076. [cited 2014 may 23].

10. Frick $S$, Uehlinger DE, Zuercher Zenklusen RM. Medical futility: predicting outcome of intensive care unit patients by nurses and doctors--a prospective comparative study. Crit Care Med. 2003 ;31(2):456-61. Available from: http://www.ncbi.nlm.nih.gov/pubmed/12576951. [cited 2014 Jun 2].

11. Villa G, De Gaudio AR, Falsini S, Lanini I, Curtis JR. Development of END-oflife ScorlNG-system to identify critically ill patients after initial critical care who are highly likely to die: a pilot study. Minerva Anestesiol Italy. 2015; 81(12):1318-28.

12. Teike Luthi F, Mabire C, Rosselet Amoussou J, Bernard M, Borasio GD, Ramelet A-S. Instruments for the identification of patients in need of palliative care: a systematic review protocol of measurement properties. Australia: JBI database Syst Rev Implement reports; 2019.

13. Hallen SAM, Ba NAMH, Mph LB, Ms CMG, Han PKJ, Mph MA. Physicians ' perceptions of the value of prognostic models : the benefits and risks of prognostic confidence; 2014. p. 1-12.

14. Curtis JR, Nielsen EL, Treece PD, Downey L, Dotolo D, Shannon SE, et al. Effect of a quality-improvement intervention on end-of-life care in the intensive care unit: a randomized trial. Am J Respir Crit Care Med. 2011; 183(3):348-55 Available from: http://www.pubmedcentral.nih.gov/ articlerender.fcgi?artid=3056230\&tool=pmcentrez\&rendertype=abstract. [cited 2014 Jun 26].

15. Penrod J, Pronovost P, Livote E, Puntillo K, Walker A, Wallenstein S, et al. Meeting standards of high-quality intensive care unit palliative care: clinical performance and predictors. Crit Care Med. 2012;40(4):1105-12.

16. Bradley $C T$, Brasel KJ. Developing guidelines that identify patients who would benefit from palliative care services in the surgical intensive care unit. Crit Care Med. 2009;37(3):946-50 Available from: http://www.ncbi.nlm. nih.gov/pubmed/19237901. [cited 2014 Jun 28].

17. Cohen SL, Sjokvist P, Lippert A, Phelan D. End-of-Life Practices in European. Units: The Ethicus Study. JAMA. 2003;290(6):790-7.

18. Sprung C, Cohen S, Sjokvist P, Baras M, Bulow H, Hovilehto S, et al. End-oflife practices in European intensive care units: the Ethicus study. JAMA. 2003;290(6):790-7.

19. DeCato TW, Engelberg RA, Downey L, Nielsen EL, Treece PD, Back AL, et al. Hospital variation and temporal trends in palliative and end-of-life care in the ICU. Crit Care Med. 2013;41(6):1405-11 Available from: http://www. pubmedcentral.nih.gov/articlerender.fcgi?artid=4009379\&tool= pmcentrez\&rendertype=abstract. [cited 2014 Jun 28].

20. Naib T, Lahewala S, Arora S, Gidwani U. Palliative care in the cardiac intensive care unit. Am J Cardiol. 2015;115(5):687-90 Available from: : https://www.ncbi.nlm.nih.gov/pubmed/25727085. 2014/12/18. United States.

21. de Barros Gulini JEHM, do Nascimento ERP, Moritz RD, de Oliveira Vargas MA, Matte DL, Cabral RP. Predictors of death in an Intensive Care Unit: contribution to the palliative approach $\Pi$ - Fatores preditores de óbito em Unidade de Terapia Intensiva: contribuição para a abordagem paliativista. Rev Esc Enferm USP. 2018;52:e03342 Available from: https://www.ncbi.nIm. nih.gov/pubmed/29947710..

22. Romano AM, Gade KE, Nielsen G, Havard R, Harrison JHJ, Barclay J, et al. Early palliative Care reduces end-of-life intensive Care unit (ICU) use but not ICU course in patients with advanced cancer. Oncologist United States. 2017;22(3):318-23.

\section{Publisher's Note}

Springer Nature remains neutral with regard to jurisdictional claims in published maps and institutional affiliations.
Ready to submit your research? Choose BMC and benefit from:

- fast, convenient online submission

- thorough peer review by experienced researchers in your field

- rapid publication on acceptance

- support for research data, including large and complex data types

- gold Open Access which fosters wider collaboration and increased citations

- maximum visibility for your research: over $100 \mathrm{M}$ website views per year

At $\mathrm{BMC}$, research is always in progress.

Learn more biomedcentral.com/submissions 